\title{
On Fast-Decodable Space-Time Block Codes
}

\author{
Ezio Biglieri, Fellow, IEEE, Yi Hong, Member, IEEE, and Emanuele Viterbo, Senior Member, IEEE
}

\begin{abstract}
We focus on full-rate, fast-decodable space-time block codes (STBCs) for $2 \times 2$ and $4 \times 2$ multiple-input multiple-output (MIMO) transmission. We first derive conditions and design criteria for reduced-complexity maximum-likelihood (ML) decodable $2 \times 2$ STBCs, and we apply them to two families of codes that were recently discovered. Next, we derive a novel reduced-complexity $4 \times 2 \mathbf{S T B C}$, and show that it outperforms all previously known codes with certain constellations.
\end{abstract}

Index Terms-Alamouti code, decoding complexity, multiple-input multiple-output (MIMO), quasi-orthogonal space-time block codes (STBCs), sphere decoder.

\section{INTRODUCTION}

$\mathbf{I}$ N 1998, Alamouti [1] invented a remarkable scheme for multiple-input multiple-output (MIMO) transmission using two transmit antennas and admitting a low-complexity maximum-likelihood (ML) decoder. Space-time block codes (STBCs) using more than two transmit antennas were designed in [2]. For such codes, ML decoding is achieved in a simple way, but, while they can achieve maximum diversity gain [3], [4], their transmission rate is reduced. The quasi-orthogonal STBCs in [5] can support a transmission rate larger than orthogonal STBCs, but at the price of a smaller diversity gain. Using algebraic number theory and cyclic division algebras, algebraic STBCs can be designed to achieve full rate and full diversity, but at the price of a higher decoding complexity.

Recently, a family of $2 \times 2$ twisted space-time transmit diversity STBCs, having full rate and full diversity, was proposed in [6]-[9]. These codes were recently rediscovered in [10], whose authors also pointed out that they enable reduced-complexity ML decoding (see infra for a definition of decoding complexity). Independently, the same codes were found in [11]. More recently, another family of full-rate, full-diversity, fast-decodable $2 \times 2$ codes for MIMO was proposed in [12].

Manuscript received August 21, 2007; revised March 17, 2008. Current version published February 04, 2009. This work was supported by the STREP project No. IST-026905 (MASCOT) within the Sixth Framework Programme of the European Commission. The work of E. Biglieri was also supported by Sequans Communications, Paris, France. The material in this paper was presented in part at the Allerton Conference on Communications Control, and Computing, Monticello, IL, September 2007 and the International Zurich Seminar on Communications, Zurich, Switzerland, March 2008.

E. Biglieri is with the Departament de Tecnologies de la Informació i les Comunicacions, Universitat Pompeu Fabra (DTIC-UPF), Barcelona, Spain (e-mail: e.biglieri@ieee.org).

Y. Hong is with the Institute of Advanced Telecom., University of Wales, Swansea, Singleton Park, SA2 8PP, U.K. (e-mail: y.hong@swansea.ac.uk).

E. Viterbo is with DEIS-Università della Calabria, 87036 Rende (CS), Italy (e-mail: viterbo@deis.unical.it).

Communicated by L. M. G. M. Tolhuizen, Associate Editor for Coding Theory.

Color versions of Figures 1 and 2 in this paper are available online at http:// ieeexplore.ieee.org.

Digital Object Identifier 10.1109/TIT.2008.2009817
Empirical evidence seems to show that the constraint of simplified ML decoding does not entail substantial performance loss. To substantiate the above claim, the present paper provides a unified view of the fast-decodable STBCs in [6]-[8], [10]-[12] for $2 \times 2$ MIMO. We show that all these codes allow the same low-complexity ML decoding procedure, which we specialize in the form of a sphere-decoder (SD) search [13]-[16]. We also derive general design criteria for full-rate, fast-decodable STBCs, and we use it to design a family of $4 \times 2$ codes based on a combination of algebraic and quasi-orthogonal structures. In this case, the full-diversity assumption is dropped in favor of simplified ML decoding. Within this family, we exhibit a code that outperforms all previously proposed $4 \times 2$ STBCs for 4-QAM signal constellation.

The balance of this paper is organized as follows. Section II introduces system model and code design criteria. In Section III, we present the concept of the fast-decodability of STBCs. In Section IV we review two families of fast-decodable $2 \times 2$ STBCs that have recently appeared in the literature, and we show how both of them enable a reduced-complexity ML decoding procedure. In Section V, we propose fast-decodable $4 \times 2$ STBCs, and we show the corresponding ML decoding complexity. Finally, conclusions are drawn in Section VI.

Notations: Boldface letters are used for column vectors, and capital boldface letters for matrices. Superscripts ${ }^{T},{ }^{\dagger}$, and ${ }^{*}$ denote transposition, Hermitian transposition, and complex conjugation, respectively. $\mathbb{Z}, \mathbb{C}$, and $\mathbb{Z}[j]$ denote the ring of rational integers, the field of complex numbers, and the ring of Gaussian integers, respectively, where $j^{2}=-1$. Also, $\boldsymbol{I}_{n}$ denotes the $n \times n$ identity matrix, and $\mathbf{0}_{m \times n}$ denotes the $m \times n$ matrix all of whose elements are 0 .

Given a complex number $x$, we define the $(\tilde{\cdot})$ operator from $\mathbb{C}$ to $\mathbb{R}^{2}$ as $\tilde{x} \triangleq[\Re(x), \Im(x)]^{T}$, where $\Re(\cdot)$ and $\Im(\cdot)$ denote real and imaginary parts. The $(\cdot)$ operator can be extended to complex vectors $\boldsymbol{x}=\left[x_{1}, \ldots x_{n}\right] \in \mathbb{C}^{n}$

$$
\tilde{\boldsymbol{x}} \triangleq\left[\Re\left(x_{1}\right), \Im\left(x_{1}\right), \ldots, \Re\left(x_{n}\right), \Im\left(x_{n}\right)\right]^{T} .
$$

Given a complex number $x$, the $(\check{.})$ operator from $\mathbb{C}$ to $\mathbb{R}^{2 \times 2}$ is defined by

$$
\check{x} \triangleq\left[\begin{array}{cc}
\Re(x) & -\Im(x) \\
\Im(x) & \Re(x)
\end{array}\right] .
$$

The $(\check{.})$ operator can be similarly extended to $n \times n$ matrices by applying it to all the entries, which yields $2 n \times 2 n$ real matrices. The following relations hold: $\tilde{A} \boldsymbol{x}=\check{\boldsymbol{A}} \tilde{\boldsymbol{x}}$ and $\boldsymbol{A}=$ $\underline{B} C \Longrightarrow \breve{A}=\breve{B} \check{C}$. Given a complex number $x$, we define the $(\overline{.})$ operator from $\mathbb{C}$ to $\mathbb{R}^{2 \times 2}$ as

$$
\bar{x} \triangleq\left[\begin{array}{cc}
-\Re(x) & -\Im(x) \\
-\Im(x) & \Re(x)
\end{array}\right] .
$$


The following relation holds:

$$
\tilde{x}^{*} \triangleq \bar{x} \cdot \tilde{y} .
$$

The $\operatorname{vec}(\cdot)$ operator stacks the $m$ column vectors of an $n \times m$ complex matrix into an $m n$ complex column vector. The $\|\cdot\|$ operation denotes the Euclidean norm of a vector. Finally, the Hermitian inner product of two complex column vectors $\boldsymbol{a}$ and $\boldsymbol{b}$ is denoted by $\langle\boldsymbol{a}, \boldsymbol{b}\rangle \triangleq \boldsymbol{a}^{T} \boldsymbol{b}^{*}$. Note also that if $\langle\boldsymbol{a}, \boldsymbol{b}\rangle=0$, then $\langle\tilde{\boldsymbol{a}}, \tilde{\boldsymbol{b}}\rangle=0$.

\section{System Model And Code Design Criteria}

We consider an $n_{r} \times n_{t}$ MIMO transmission over a blockfading channel. The received signal matrix $Y \in \mathbb{C}^{n_{r} \times T}$ is

$$
\boldsymbol{Y}=\boldsymbol{H} \boldsymbol{X}+\boldsymbol{N}
$$

where $\boldsymbol{X} \in \mathbb{C}^{n_{t} \times T}$ is the codeword matrix, transmitted over $T$ channel uses. Moreover, $\boldsymbol{N} \in \mathbb{C}^{n_{r} \times T}$ is a complex white Gaussian noise with independent and identically distributed (i.i.d.) entries $\sim \mathcal{N}_{\mathbb{C}}\left(0, N_{0}\right)$, and $\boldsymbol{H}=\left[h_{i \ell}\right] \in \mathbb{C}^{n_{r} \times n_{t}}$ is the channel matrix, assumed to remain constant during the transmission of a codeword, and to take on independent values from codeword to codeword. The elements of $H$ are assumed to be i.i.d. circularly symmetric Gaussian random variables $\sim \mathcal{N}_{\mathbb{C}}(0,1)$. The realization of $\boldsymbol{H}$ is assumed to be known at the receiver, but not at the transmitter. The following definitions are relevant here.

Definition 1: (Code Rate): Let $\kappa$ be the number of independent information symbols per codeword, drawn from a complex constellation $\mathcal{S}$. The code rate of an STBC is defined as $R=\kappa / T$ symbols per channel use. If $\kappa=n_{r} T$, the STBC is said to have full rate.

Consider ML decoding. This consists of finding the code matrix that achieves the minimum of the squared Frobenius norm $m(\boldsymbol{X}) \triangleq\|\boldsymbol{Y}-\boldsymbol{H} \boldsymbol{X}\|^{2}$.

Definition 2: (Decoding Complexity): The ML decoding complexity is defined as the minimum number of values of $m(\boldsymbol{X})$ that should be computed in ML decoding. This number cannot exceed $M^{\kappa}$, with $M=|\mathcal{S}|$, the complexity of the exhaustive-search ML decoder.

Consider two codewords $\boldsymbol{X}$ and $\hat{\boldsymbol{X}} \neq \boldsymbol{X}$. Let $r$ denote the minimum rank of the matrix $\boldsymbol{X}-\hat{\boldsymbol{X}}$, and $\delta$ the product distance, i.e., the product of nonzero eigenvalues of the codeword distance matrix $\boldsymbol{E} \triangleq(\boldsymbol{X}-\hat{\boldsymbol{X}})(\boldsymbol{X}-\hat{\boldsymbol{X}})^{\dagger}$. The error probability of an STBC is upper-bounded by the following union bound:

$$
P(e) \leq \frac{1}{M^{\kappa}} \sum_{r} \sum_{\delta} A(r, \delta) P(r, \delta)
$$

where $P(r, \delta)$ denotes the pairwise error probability (PEP) of the codeword differences with rank $r$ and product distance $\delta$, and $A(r, \delta)$ the associated multiplicity. In [3], the "rank-anddeterminant criterion" (RDC) was proposed to maximize both the minimum rank $r$ and the minimum determinant

$$
\delta_{\min } \triangleq \min _{\boldsymbol{X} \neq \hat{\boldsymbol{X}}} \operatorname{det}(\boldsymbol{E}) .
$$

For a full-diversity STBC, i.e., $r=n_{t}$ for all $\boldsymbol{E}$ matrices, this criterion yields diversity gain $n_{r} n_{t}$ and coding gain $\left(\delta_{\min }\right)^{1 / n_{t}}[3]$. For STBC with $\delta_{\min }=0$, and hence without full diversity, one should minimize $A(r, \delta)$ with $r \leq n_{t}$.

\section{A. Linear Codes, and Codes With the Alamouti Structure}

Linear STBCs are especially relevant in our context, because they admit ML sphere decoding.

Definition 3: (Linear STBC): A STBC carrying $\kappa$ symbols $\boldsymbol{s}=\left[s_{1}, \ldots, s_{\kappa}\right]$ is said to be (real) linear if we can write $\operatorname{vec}(\boldsymbol{X})=\mathbb{G} \tilde{\boldsymbol{s}}$ for some $\mathbb{G} \in \mathbb{R}^{2 n_{t} T \times 2 \kappa}$. The matrix $\mathbb{G}$ is called the (real) generator matrix of the linear code. If a complex matrix $\boldsymbol{G} \in \mathbb{C}^{n_{t} T \times \kappa}$ exists such that $\mathbb{G}=\breve{G}$, then we can write $\operatorname{vec}(\boldsymbol{X})=\boldsymbol{G s}$ which identifies a complex linear STBC, with $\boldsymbol{G}$ its complex generator matrix.

Definition 4: (Cubic Shaping): For a linear STBC, if its real generator matrix $\mathbb{G}$ is an orthogonal matrix satisfying $\mathbb{G}^{\dagger} \mathbb{G}=$ $\boldsymbol{I}_{2 \kappa}$, then we say that the STBC has cubic shaping (see [17] for the significance of cubic shaping).

Linear STBCS admit the canonical decomposition

$$
\boldsymbol{X}=\sum_{\ell=1}^{\kappa}\left(a_{\ell} \boldsymbol{A}_{\ell}+j b_{\ell} \boldsymbol{B}_{\ell}\right)
$$

where $a_{\ell}$ and $b_{\ell}$ are the real and imaginary parts of $s_{\ell}$, respectively, and $\boldsymbol{A}_{\ell}, \boldsymbol{B}_{\ell}, \ell=1, \ldots, \kappa$ are $n_{t} \times T$ (generally complex) matrices. With this decomposition, (1) can be rewritten using only real quantities

$$
\widetilde{\operatorname{vec}(\boldsymbol{Y})}=\mathbb{F} \tilde{\boldsymbol{s}}+\widetilde{\operatorname{vec}(\boldsymbol{N})}
$$

where

$$
\begin{array}{r}
\left.\mathbb{F} \triangleq\left[\operatorname{vec} \widetilde{\boldsymbol{H}} A_{1}\right), \operatorname{vec} \widetilde{\boldsymbol{H}} \boldsymbol{B}_{1}\right), \ldots, \operatorname{vec} \widetilde{\left.\left.\boldsymbol{H} \boldsymbol{B}_{\kappa}\right)\right]} \\
=\operatorname{diag}(\check{\boldsymbol{H}}, \ldots, \check{\boldsymbol{H}}) \mathbb{G}
\end{array}
$$

and $\mathbb{G}=\left[\widetilde{\operatorname{vec}\left(\boldsymbol{A}_{1}\right)}, \ldots, \operatorname{vec(\boldsymbol {B}_{\kappa })}\right]$. Note that the $\mathbb{F}$ matrix depends on $\boldsymbol{H}$. With complex linear STBC, we may use only complex quantities

$$
\operatorname{vec}(\boldsymbol{Y})=\boldsymbol{F s}+\operatorname{vec}(\boldsymbol{N})
$$

where now

$$
\begin{aligned}
\boldsymbol{F} \triangleq\left[\operatorname{vec}\left(\boldsymbol{H} \boldsymbol{A}_{1}\right), \operatorname{vec}\left(\boldsymbol{H} \boldsymbol{B}_{1}\right), \ldots, \operatorname{vec}\left(\boldsymbol{H} \boldsymbol{B}_{\kappa}\right)\right] \\
=\operatorname{diag}(\boldsymbol{H}, \ldots, \boldsymbol{H}) \boldsymbol{G}
\end{aligned}
$$

with $\boldsymbol{G}=\left[\operatorname{vec}\left(\boldsymbol{A}_{1}\right), \ldots, \operatorname{vec}\left(\boldsymbol{B}_{\kappa}\right)\right], \check{\boldsymbol{G}}=\mathbb{G}$, and $\check{\boldsymbol{F}}=\mathbb{F}$.

Definition 5: (Alamouti Structure): We say that an STBC has the Alamouti structure if

$$
\boldsymbol{X}=\left[\begin{array}{rr}
\alpha s_{1} & -\beta s_{2}^{*} \\
\alpha s_{2} & \beta s_{1}^{*}
\end{array}\right]
$$

where $s_{i} \in \mathbb{C}$ with $i=1,2$, and $\alpha, \beta \in \mathbb{C},|\alpha|^{2}=|\beta|^{2}$, and $|\alpha|^{2}+|\beta|^{2}=1$. 
From the definition of linear codes, we have

$$
\mathbb{G}=\left[\begin{array}{cc}
\check{\alpha} & \check{0} \\
\check{0} & \check{\alpha} \\
\check{0} & \check{\beta} \\
-\check{\beta} & \check{0}
\end{array}\right]
$$

and can see, by direct calculation, that $\mathbb{G}^{T} \mathbb{G}=I_{4}$, which implies the cubic shaping of these STBCs. Moreover, given $\boldsymbol{H}=\left[h_{i j}\right] \in \mathbb{C}^{2 \times 2}$ and $\boldsymbol{Y}=\left[y_{i j}\right] \in \mathbb{C}^{2 \times 2}$, let us define

$$
\boldsymbol{y} \triangleq\left[y_{11}, y_{21}, y_{12}^{*}, y_{22}^{*}\right]^{T} \quad \boldsymbol{n} \triangleq\left[n_{11}, n_{21}, n_{12}^{*}, n_{22}^{*}\right]^{T}
$$

where the last two elements of the vectorized matrices are conjugated. We can write (1) as

$$
\boldsymbol{y}=\boldsymbol{F}^{(*)} \boldsymbol{s}+\boldsymbol{n}
$$

where

$$
\boldsymbol{F}^{(*)} \triangleq\left[\boldsymbol{f}_{1} \mid \boldsymbol{f}_{2}\right]=\left[\begin{array}{cc}
\alpha h_{11} & \alpha h_{12} \\
\alpha h_{21} & \alpha h_{22} \\
\beta^{*} h_{12}^{*} & -\beta^{*} h_{11}^{*} \\
\beta^{*} h_{22}^{*} & -\beta^{*} h_{21}^{*}
\end{array}\right]
$$

and

$$
\mathbb{F} \triangleq \check{\boldsymbol{F}}^{(*)}=\left[\check{\boldsymbol{f}}_{1} \mid \check{\boldsymbol{f}}_{2}\right] .
$$

Note that $\boldsymbol{F}^{(*)}$ has its last two rows conjugated. In complex notations, multiplication of $\boldsymbol{y}$ at the receiver by $\left(\boldsymbol{F}^{(*)}\right)^{\dagger}$ is equivalent to matched filtering. Direct calculation shows that, for codes with the Alamouti structure

$$
\boldsymbol{F}^{(*)^{\dagger}} \boldsymbol{F}^{(*)}=\left(\sum_{i} \sum_{j}\left|h_{i j}\right|^{2}\right) \boldsymbol{I}_{2}, \quad \text { i.e., }\left\langle\boldsymbol{f}_{1}, \boldsymbol{f}_{2}\right\rangle=0
$$

and hence ML decoding can be done symbol-by-symbol, which, under our definition, yields complexity $2 M$.

\section{FASt Decoding With QR Decomposition}

Consider a linear STBC carrying $\kappa$ independent quadrature amplitude modeulation (QAM) information symbols. Following (5), at the receiver, the SD algorithm can be used to conduct ML decoding based on QR decomposition of matrix $\boldsymbol{F}[16]: F=\boldsymbol{Q R}$, where $Q \in \mathbb{C}^{\kappa \times \kappa}$ is unitary, and $R \in \mathbb{C}^{\kappa \times \kappa}$ is upper-triangular. The ML decoder minimizes $\left\|\boldsymbol{Q}^{\dagger} \operatorname{vec}(\boldsymbol{Y})-\boldsymbol{R} \boldsymbol{s}\right\|$. If we write

$$
\boldsymbol{F}=\left[\boldsymbol{f}_{1}\left|\boldsymbol{f}_{2}\right| \cdots \mid \boldsymbol{f}_{\kappa}\right] \in \mathbb{C}^{\kappa \times \kappa}
$$

then the matrices $Q$ and $R$ have the general form

$$
\boldsymbol{Q}=\left[\boldsymbol{e}_{1}\left|\boldsymbol{e}_{2}\right| \cdots \mid \boldsymbol{e}_{\kappa}\right]
$$

and

$$
\boldsymbol{R}=\left[\begin{array}{cccc}
\left\|\boldsymbol{d}_{1}\right\| & \left\langle\boldsymbol{f}_{2}, \boldsymbol{e}_{1}\right\rangle & \cdots & \left\langle\boldsymbol{f}_{\kappa}, \boldsymbol{e}_{1}\right\rangle \\
0 & \left\|\boldsymbol{d}_{2}\right\| & \ddots & \left\langle\boldsymbol{f}_{\kappa}, \boldsymbol{e}_{2}\right\rangle \\
0 & 0 & \ddots & \vdots \\
0 & 0 & 0 & \left\|\boldsymbol{d}_{\kappa}\right\|
\end{array}\right]
$$

where

$$
\begin{array}{ll}
\boldsymbol{d}_{1}=f_{1} & \boldsymbol{e}_{1}=\frac{\boldsymbol{d}_{1}}{\left\|\boldsymbol{d}_{1}\right\|}=\frac{f_{1}}{\left\|f_{1}\right\|} \\
\boldsymbol{d}_{i}=f_{i}-\sum_{j=1}^{i-1} \operatorname{Proj}_{\boldsymbol{e}_{j}} \boldsymbol{f}_{i} & \boldsymbol{e}_{i}=\frac{\boldsymbol{d}_{i}}{\left\|\boldsymbol{d}_{i}\right\|}, \quad i=2, \ldots, \kappa
\end{array}
$$

and $\operatorname{Proj}_{\boldsymbol{u}} \boldsymbol{v} \triangleq\langle\boldsymbol{v}, \boldsymbol{u}\rangle /\langle\boldsymbol{u}, \boldsymbol{u}\rangle \boldsymbol{u}$. This formulation of the QR decomposition coincides with the Gram-Schmidt procedure applied to the column vectors of $\boldsymbol{F}$. It was pointed out in [16] that the search procedure of an SD can be visualized as a bounded tree search. If a standard SD is used for the above STBC, we have $\kappa$ levels of the complex SD tree, where the worst case computation complexity is $M^{\kappa}$. However, zeros appearing among the entries of $\boldsymbol{R}$ can lead to simplified SD, as discussed in the following.

If the condition

$$
\left\langle\boldsymbol{f}_{2}, \boldsymbol{e}_{i}\right\rangle=\left\langle\boldsymbol{f}_{3}, \boldsymbol{e}_{i}\right\rangle=\cdots=\left\langle\boldsymbol{f}_{k^{\prime}}, \boldsymbol{e}_{i}\right\rangle=0
$$

is satisfied for $i=1, \ldots, k^{\prime}-1$ and for some $k^{\prime} \leq \kappa$, then $k^{\prime}$ levels can be removed from the complex SD tree, and we can employ a $\left(\kappa-k^{\prime}\right)$-dimensional complex SD. In it, we first estimate the partial vector $\left(s_{k^{\prime}+1}, \ldots, s_{\kappa}\right)$. For every such vector (there are $M^{\kappa-k^{\prime}}$ of them), a linear ML decoding, of complexity $k^{\prime} M$ is used to choose $s_{1}, \ldots, s_{k^{\prime}}$ so as to minimize the total ML metric. Hence, the worst case decoding complexity is $k^{\prime} M^{\left(\kappa-k^{\prime}+1\right)}$. The components $s_{i}$ should be sorted in order to maximize $k^{\prime}$.

Analysis of the structure of the matrix $\boldsymbol{R}$ yields the following observation:

Zero entries of $\boldsymbol{R}$, besides those in (13), lead to faster metric computations in the relevant SD branches, but not to a reduction of the number of branches. We conclude this section with the following definition.

Definition 6: (Fast-Decodable STBCs): A linear STBC allows fast ML decoding if (13) is satisfied, yielding a complexity of the order of $k^{\prime} M^{\kappa-k^{\prime}+1}$.

\section{FAST-DeCODABLE CODES FOR $2 \times 2$ MIMO, AND ML DECODING}

Consider now full-rate $(R=2)$ and full-diversity fast-decodable $2 \times 2$ STBCs, i.e., with $\kappa=4$ symbols/codeword and $r=$ $n_{t}$. Here we examine two families of $2 \times 2$ full-rate, full-diversity fast-decodable STBCs, endowed with the following structure:

$$
\boldsymbol{X}=\boldsymbol{X}_{1,2}\left(s_{1}, s_{2}\right)+\boldsymbol{X}_{3,4}\left(s_{3}, s_{4}\right)
$$

where the first (resp., second) component code encodes symbols $s_{1}, s_{2}$ (resp., $s_{3}, s_{4}$ ).

Family I: In this family of fast-decodable STBCs, independently derived in [7], [10], [11], $\boldsymbol{X}_{1,2}\left(s_{1}, s_{2}\right)$ has the Alamouti structure [1] with $\alpha=\beta=1$ and $\boldsymbol{X}_{3,4}\left(s_{3}, s_{4}\right)$ is chosen as follows: let

$$
\boldsymbol{T} \triangleq\left[\begin{array}{cc}
1 & 0 \\
0 & -1
\end{array}\right] \text { and }\left[\begin{array}{l}
z_{1} \\
z_{2}
\end{array}\right]=\boldsymbol{U}\left[\begin{array}{l}
s_{3} \\
s_{4}
\end{array}\right]
$$

where $z_{1}, z_{2} \in \mathbb{C}$ and $\boldsymbol{U} \in \mathbb{C}^{2 \times 2}$ is the unitary matrix

$$
\boldsymbol{U}=\left[\begin{array}{cc}
\varphi_{1} & -\varphi_{2}^{*} \\
\varphi_{2} & \varphi_{1}^{*}
\end{array}\right]
$$

with $\left|\varphi_{1}\right|^{2}+\left|\varphi_{2}\right|^{2}=1$. We have

$$
\begin{aligned}
& \boldsymbol{X}_{3,4}\left(s_{3}, s_{4}\right)=\boldsymbol{T}\left[\begin{array}{cc}
z_{1} & -z_{2}^{*} \\
z_{2} & z_{1}^{*}
\end{array}\right] \\
& =\boldsymbol{T}\left[\begin{array}{cc}
\varphi_{1} s_{3}-\varphi_{2}^{*} s_{4} & -\left(\varphi_{2} s_{3}+\varphi_{1}^{*} s_{4}\right)^{*} \\
\varphi_{2} s_{3}+\varphi_{1}^{*} s_{4} & \left(\varphi_{1} s_{3}-\varphi_{2}^{*} s_{4}\right)^{*}
\end{array}\right]
\end{aligned}
$$


which has the Alamouti structure (7). Vectorizing, and separating real and imaginary parts of the matrix $\boldsymbol{X}$, we obtain

$$
\widetilde{\operatorname{vec}(\boldsymbol{X})}=\mathbb{G}\left[\tilde{s}_{1}, \tilde{s}_{2}, \tilde{s}_{3}, \tilde{s}_{4}\right]^{T}=\mathbb{G}_{1}\left[\tilde{s}_{1}, \tilde{s}_{2}\right]^{T}+\mathbb{G}_{2}\left[\tilde{s}_{3}, \tilde{s}_{4}\right]^{T} .
$$

Thus, $\mathbb{G}=\left[\mathbb{G}_{1} \mid \mathbb{G}_{2}\right] \in \mathbb{R}^{8 \times 8}$ is the generator matrix of the code. Specifically, $\mathbb{G}_{1} \in \mathbb{R}^{8 \times 4}$ is the generator matrix of $\boldsymbol{X}_{1,2}$, and $\mathbb{G}_{2} \in \mathbb{R}^{8 \times 4}$ is the generator matrix of $\boldsymbol{X}_{3,4}$. The matrix $\mathbb{G}_{1}$ has the structure of (8) with coefficients $\alpha_{1,2}$ and $\beta_{1,2}$

$$
\mathbb{G}_{1} \triangleq\left[\boldsymbol{g}_{1}\left|\boldsymbol{g}_{2}\right| \boldsymbol{g}_{3} \mid \boldsymbol{g}_{4}\right] \triangleq\left[\begin{array}{cc}
\check{\alpha}_{1,2} & \check{0}^{\circ} \\
\check{0} & \check{\alpha}_{1,2} \\
\check{\check{\beta}}_{1,2} \\
-\bar{\beta}_{1,2} & \check{0}
\end{array}\right]
$$

and

$$
\mathbb{G}_{2} \triangleq\left[\boldsymbol{g}_{5}\left|\boldsymbol{g}_{6}\right| \boldsymbol{g}_{7} \mid \boldsymbol{g}_{8}\right] \triangleq\left[\begin{array}{rr}
\check{\varphi}_{1} & -\check{\varphi}_{2}^{*} \\
-\check{\varphi}_{2} & -\breve{\varphi}_{1}^{*} \\
\bar{\varphi}_{2}^{*} & \bar{\varphi}_{1} \\
\bar{\varphi}_{1}^{*} & -\bar{\varphi}_{2}
\end{array}\right]
$$

Direct computation shows the following.

Property 1 (Column Orthogonality): Both $\mathbb{G}_{1}$ and $\mathbb{G}_{2}$ have orthogonal columns: $\left\langle\boldsymbol{g}_{i}, \boldsymbol{g}_{j}\right\rangle=0$, where $i, j \in[1,4]$ or $i, j \in$ $[5,8]$, i.e., $\mathbb{G}_{1}^{\dagger} \mathbb{G}_{1}=\mathbb{G}_{2}{ }^{\dagger} \mathbb{G}_{2}=\boldsymbol{I}_{4}$.

Property 2 (Mutual Column Orthogonality and Cubic Shaping): With $\boldsymbol{T}$ as in (15), the subspace spanned by the columns of $\mathbb{G}_{2}$ is orthogonal to the one spanned by the columns of $\mathbb{G}_{1}$, i.e., $\left\langle\boldsymbol{g}_{i}, \boldsymbol{g}_{j}\right\rangle=0$, for $i \in[1,4]$ and $j \in[5,8]$. Since $\mathbb{G}=\left[\mathbb{G}_{1} \mid \mathbb{G}_{2}\right]$, we have

$$
\mathbb{G}^{\dagger} \mathbb{G}=\left[\begin{array}{cc}
\mathbb{G}_{1}^{\dagger} \mathbb{G}_{1} & \mathbf{0} \\
\mathbf{0} & \mathbb{G}_{2}^{\dagger} \mathbb{G}_{2}
\end{array}\right]=\boldsymbol{I}_{8}
$$

This implies cubic shaping [17].

The matrix $\boldsymbol{U}$ should be chosen so as to achieve full rank and maximize the minimum determinant. The best known code of the form (14) was first found in [7], and independently rediscovered in [10] and [11] by numerical optimization.

Family II: In the second family of fast-decodable STBCs [12], both $\boldsymbol{X}_{1,2}\left(s_{1}, s_{2}\right)$ and $\boldsymbol{X}_{3,4}\left(s_{3}, s_{4}\right)$, have the Alamouti structure (7), with coefficients $\alpha_{1,2}, \beta_{1,2}$ used for $\boldsymbol{X}_{1,2}\left(s_{1}, s_{2}\right)$, and $\alpha_{3,4}, \beta_{3,4}$ for $\boldsymbol{X}_{3,4}\left(s_{3}, s_{4}\right)$. The only difference between Family II and Family I is that Family II codes do not satisfy Property 2: $\mathbb{G}$ is not an orthogonal matrix, and hence codes in this family do not exhibit cubic shaping.

Table I compares the minimum determinant $\delta_{\min }$ of the best known STBCs in the two families with that of the Golden code [18] for 4-, 16-, and 64-QAM signaling. In our computations, we assume that the constellation points have odd-integer coordinates. It can be seen that the minimum determinant of Family I STBCs and of the Golden code [18] are constant across constellations, while the minimum determinant of Family II STBC decreases slowly as the size of the signal constellation increases. The codes of [7], [10], [11] exhibit a minimum determinant slightly larger than those of [12].

Let us define the signal-to-noise ratio SNR $\triangleq n_{t} E_{s} / N_{0}$, where $E_{s}$ the average energy. Fig. 1 compares the codeword
TABLE I

The Minimum Determinants $\delta_{\min }$ OF THE Golden Code AND Two FAMILIES OF FAST-DECODABLE STBCS WITH 4-, 16-, AND 64-QAM SIGNALING

\begin{tabular}{|c|c|c|c|}
\hline $\mathcal{G}$ & $\delta_{\min }$, 4-QAM & $\delta_{\min }, 16-\mathrm{QAM}$ & $\delta_{\min }$, 64-QAM \\
\hline 1st Family & 2.2857 & 2.2857 & 2.2857 \\
\hline 2nd Family & 1.9973 & 1.9796 & 1.8784 \\
\hline Golden Code & 3.2 & 3.2 & 3.2 \\
\hline
\end{tabular}

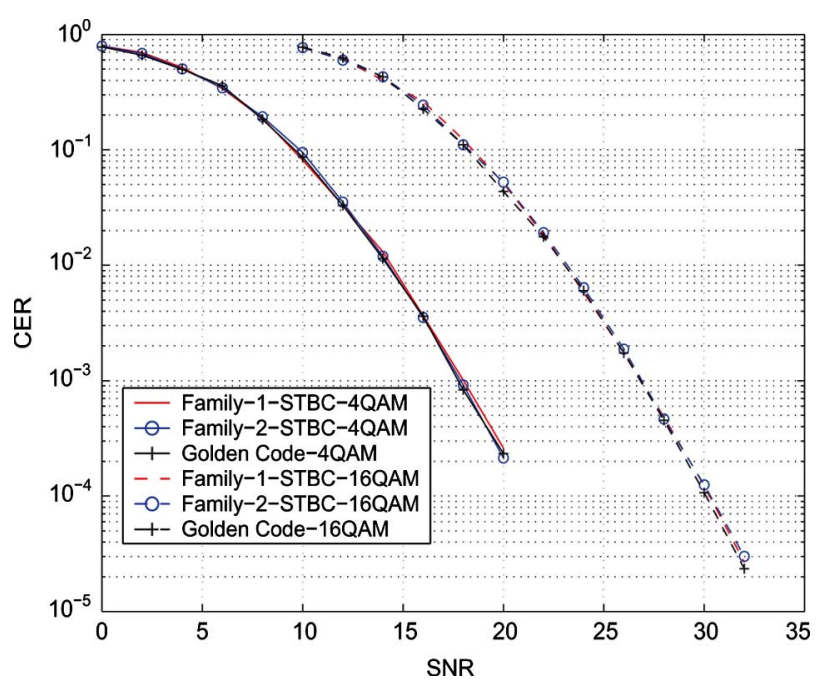

Fig. 1. Comparison of the CER of the best $2 \times 2$ codes in two fast-decodable STBC families and of the Golden code with 4- and 16-QAM signaling.

error rate (CER) of the best STBCs in the two families and of the Golden code with 4- and 16-QAM signaling. It is shown that both families of fast-decodable STBCs exhibit similar CER performances, and both differ slightly, at high SNR, from that of Golden code. Since the latter has the best CER known, but does not admit simplified decoding, this small difference can be viewed as the penalty to be paid for complexity reduction.

\section{A. Decoding Family I and Family II STBCs}

By direct computation, we have $\left\langle\boldsymbol{f}_{2}, \boldsymbol{e}_{1}\right\rangle=0$ and $\left\langle\boldsymbol{f}_{4}, \boldsymbol{e}_{3}\right\rangle=$ 0 . In fact, we can see that the full-rate fast-decodable STBCs are obtained by linearly combining two rate- 1 codes: $\boldsymbol{X}_{1,2}$ and $\boldsymbol{X}_{3,4}$. Moreover, by examining the structures of the $2 \times 2$ STBCs and the matrix $\boldsymbol{R}$, we obtain the results that follow.

Proposition 1: We have $\left\langle\boldsymbol{f}_{2}, \boldsymbol{e}_{1}\right\rangle=0$ if and only if $\boldsymbol{X}_{1,2}$ is an Alamouti STBC. Consequently, the fast-decodable full-rate $2 \times 2$ STBCs only exist for $k^{\prime}=2$ and their corresponding worst case decoding complexity does not exceed $2 M^{3}$.

Proof: First, if $\boldsymbol{X}_{1,2}$ is an Alamouti STBC, from (12) we conclude that $\left\langle f_{2}, f_{1}\right\rangle=0$, and therefore

$$
\left\langle\boldsymbol{f}_{2}, \boldsymbol{e}_{1}\right\rangle=\left\langle\boldsymbol{f}_{2}, \frac{\boldsymbol{f}_{1}}{\left\|\boldsymbol{f}_{1}\right\|}\right\rangle=0 .
$$

Second, since $\boldsymbol{X}_{1,2}$ is a rate- 1 STBC, it was shown in [2, Theorem 5.4.2] that complex linear-processing orthogonal designs only exist in two dimensions and the Alamouti scheme is unique. Thus, 1) the orthogonality condition $\left\langle\boldsymbol{f}_{2}, \boldsymbol{e}_{1}\right\rangle=0$ in $2 \times 2$ STBCs implies that $\boldsymbol{X}_{1,2}$ must have an Alamouti structure, which completes the proof of the converse implication; and 2) this also implies that it is only possible to have $\left\langle\boldsymbol{f}_{2}, \boldsymbol{e}_{1}\right\rangle=0$ for 
the fast-decodable full-rate $2 \times 2$ STBCs. Based on Definition 6 , it yields $k^{\prime}=2$ and the worst-case decoding complexity of $2 M^{3}$.

To further save computational complexity, we may require $\left\langle\boldsymbol{f}_{4}, \boldsymbol{e}_{3}\right\rangle=0$. This can be obtained if both $\boldsymbol{X}_{1,2}$ and $\boldsymbol{X}_{3,4}$ have the Alamouti structure. Note that this condition is sufficient but not necessary, since the Alamouti structure implies $\left\langle\boldsymbol{f}_{4}, \boldsymbol{e}_{3}\right\rangle=$ 0 , but the converse is not true.

The Alamouti structure of $\boldsymbol{X}_{1,2}$ and $\boldsymbol{X}_{3,4}$ yields some zero entries in matrix $\boldsymbol{R}$ and we have the following proposition.

Proposition 2: The other elements in the matrix $\boldsymbol{R}$ cannot be nulled.

Proof: By direct computation we easily verify $\left\langle\boldsymbol{f}_{i}, \boldsymbol{f}_{j}\right\rangle \neq$ $0, i \in[1,2], j \in[3,4]$. Therefore, this code is not an orthogonal STBC [2], and we have

$$
\left\langle\boldsymbol{f}_{3}, \boldsymbol{e}_{1}\right\rangle=\left\langle\boldsymbol{f}_{3}, \frac{\boldsymbol{f}_{1}}{\left\|\boldsymbol{f}_{1}\right\|}\right\rangle \neq 0 \text { and }\left\langle\boldsymbol{f}_{4}, \boldsymbol{e}_{1}\right\rangle \neq 0 .
$$

With $\left\langle\boldsymbol{f}_{2}, \boldsymbol{e}_{1}\right\rangle=0$, we have

$$
\boldsymbol{e}_{2}=\frac{\boldsymbol{f}_{2}-\operatorname{Proj}_{\boldsymbol{e}_{1}} \boldsymbol{f}_{2}}{\left\|\boldsymbol{f}_{2}-\operatorname{Proj}_{\boldsymbol{e}_{1}} \boldsymbol{f}_{2}\right\|}=\frac{\boldsymbol{f}_{2}}{\left\|\boldsymbol{f}_{2}\right\|}
$$

then

$$
\left\langle\boldsymbol{f}_{3}, \boldsymbol{e}_{2}\right\rangle=\left\langle\boldsymbol{f}_{3}, \frac{\boldsymbol{f}_{2}}{\left\|\boldsymbol{f}_{2}\right\|}\right\rangle \neq 0 \text { and }\left\langle\boldsymbol{f}_{4}, \boldsymbol{e}_{2}\right\rangle \neq 0 .
$$

Due to (19) and (21), the corresponding elements in $\boldsymbol{R}$ cannot be nulled.

In summary, a $2 \times 2 \mathrm{STBC}$ of the form (14) has complexity $2 M^{3}$ if it satisfies Proposition 1 . If in addition $\boldsymbol{X}_{3,4}$ has Alamouti structure, then extra computational savings are available in the SD algorithm. Moreover, if cubic shaping is required, the generator matrix $\mathbb{G}$ of the STBC is orthogonal.

\section{NEW $4 \times 2$ STBC AND ITS DECODING COMPLEXITY}

Here we design a fast-decodable full-rate $(R=2) 4 \times 2$ STBC based on the concepts elaborated upon in the previous sections. Specifically, using the twisted structure described above, we combine linearly two rate- 1 codes. Since rate- 1 orthogonal codes do not exists for four transmit antennas, we resort quasi-orthogonal STBCs instead [5].

Definition 7 (Quasi-Orthogonal Structure): [5] A code whose words have the form

$$
\boldsymbol{X}=\left[\begin{array}{rrrr}
s_{1} & -s_{2}^{*} & -s_{3}^{*} & s_{4} \\
s_{2} & s_{1}^{*} & -s_{4}^{*} & -s_{3} \\
s_{3} & -s_{4}^{*} & s_{1}^{*} & -s_{2} \\
s_{4} & s_{3}^{*} & s_{2}^{*} & s_{1}
\end{array}\right]
$$

or another equivalent form as defined in [5], where $s_{i} \in \mathbb{C}$, $i=1, \ldots, 4$, is said to have a quasi-orthogonal structure. The quasi-orthogonal STBC is not full rank and has $r=2$.

Definition 8 (Full-Rate, Fast-Decodable STBC for $4 \times 2$ MIMO): A full-rate $(R=2)$, fast-decodable STBC for $4 \times 2$ MIMO, denoted $\mathcal{G}^{\prime}$, has $\kappa=8$ symbols/codeword, and can be decoded by a 12-dimensional real SD algorithm (rather than the standard 16-dimensional SD).

The $4 \times 4$ codeword matrix $\boldsymbol{X} \in \mathcal{G}^{\prime}$ encodes eight QAM symbols $\boldsymbol{s}=\left[s_{1}, \ldots, s_{8}\right] \in \mathbb{Z}^{8}[j]$, and is transmitted by using the channel four times, so that $T=4$. We admit the sum structure

$$
\boldsymbol{X}=\boldsymbol{X}_{1,2}\left(s_{1}, s_{2}, s_{3}, s_{4}\right)+\boldsymbol{X}_{3,4}\left(s_{5}, s_{6}, s_{7}, s_{8}\right)
$$

where $\boldsymbol{X}_{1,2}\left(s_{1}, s_{2}, s_{3}, s_{4}\right)$ is a quasi-orthogonal STBC, and

$$
\boldsymbol{X}_{3,4}\left(z_{1}, z_{2}, z_{3}, z_{4}\right) \triangleq \boldsymbol{T}\left[\begin{array}{rrrr}
z_{1} & -z_{2}^{*} & -z_{3}^{*} & z_{4} \\
z_{2} & z_{1}^{*} & -z_{4}^{*} & -z_{3} \\
z_{3} & -z_{4}^{*} & z_{1}^{*} & -z_{2} \\
z_{4} & z_{3}^{*} & z_{2}^{*} & z_{1}
\end{array}\right]
$$

with

$$
\boldsymbol{T}=\left[\begin{array}{rrrr}
1 & 0 & 0 & 0 \\
0 & 1 & 0 & 0 \\
0 & 0 & -1 & 0 \\
0 & 0 & 0 & -1
\end{array}\right]
$$

and

$$
\left[z_{1}, z_{2}, z_{3}, z_{4}\right]^{T}=\boldsymbol{U}\left[s_{5}, s_{6}, s_{7}, s_{8}\right]^{T}
$$

where $z_{i} \in \mathbb{C}, i=1, \ldots, 4, s_{k} \in \mathbb{Z}[j], k=5, \ldots, 8$, and $\boldsymbol{U}$ is a $4 \times 4$ unitary matrix.

Remark 1 (Rank 2): Since the matrix $\boldsymbol{X}_{1,2}$ has the quasi-orthogonal structure, the code does not have full rank. In particular, it has $r=2$.

Remark 2 (Cubic Shaping): Direct computation shows that the matrix $\boldsymbol{T}$ guarantees cubic shaping.

We conduct a search over the matrices $\boldsymbol{U}$, leading to the minimum of $\sum_{\delta} A(2, \delta)$, where the terms $A(2, \delta)$ represent the total number of pairwise error events of rank 2 and product distance $\delta$. Since an exhaustive search through all $4 \times 4$ unitary matrices is too complex, we focus on those with the form

$$
U=D P
$$

where $\boldsymbol{P} \triangleq[\exp (j 2 \pi \ell n / 4)]$ is a $4 \times 4$ discrete Fourier transform matrix, $\boldsymbol{D}=\operatorname{diag}\left(\exp \left(j 2 \pi n_{\ell} / N\right)\right)$ for some integer $N$, and $n_{\ell} \in\{0,1, \ldots, N\}$ for $\ell=1, \ldots, 4$.

For 4-QAM signaling, taking $N=7$ and $n_{\ell}=1,2,5,6$, we have obtained $\boldsymbol{U}$ as

$$
\left[\begin{array}{rrrr}
0.31+0.39 i & 0.31+0.39 i & 0.31+0.39 i & 0.31+0.39 i \\
-0.11+0.49 i & -0.49-0.11 i & 0.11-0.49 i & 0.49+0.11 i \\
-0.11-0.49 i & 0.11+0.49 i & -0.11-0.49 i & 0.11+0.49 i \\
0.31-0.39 i & -0.39-0.31 i & -0.31+0.39 i & 0.39+0.31 i
\end{array}\right]
$$

which yields the minimum $\sum_{\delta} A(2, \delta)$.

Under 4-QAM signaling, we compare the minimum determinants $\delta_{\min }$ and their associated multiplicities $A\left(r, \delta_{\min }\right)$, as well as the CERs of the above STBC to the following $4 \times 2$ codes.

1) Code with the structure (22), with $\boldsymbol{U}$ the $4 \times 4$ "perfect" rotation matrix [19].

2) The best DjABBA code of [8].

3) The "perfect" two-layer code of [20]. 
TABLE II

MinIMUM DETERMINANTS OF $4 \times 2$ STBCS With 4-QAM SignALING

\begin{tabular}{|c|c|c|}
\hline Codes & $\delta_{\min }$ & Multiplicities \\
\hline \hline New STBC & 0 & $\sum_{\delta} A(2, \delta)=160$ \\
\hline \hline Perfect Code U matrix & 0 & $\sum_{\delta} A(2, \delta)=560$ \\
\hline \hline DjABBA & 0.8304 & $A(4,0.8304)=770$ \\
\hline \hline Two-Layers Perfect Code & 0.0016 & $A(4,0.0016)=128$ \\
\hline
\end{tabular}

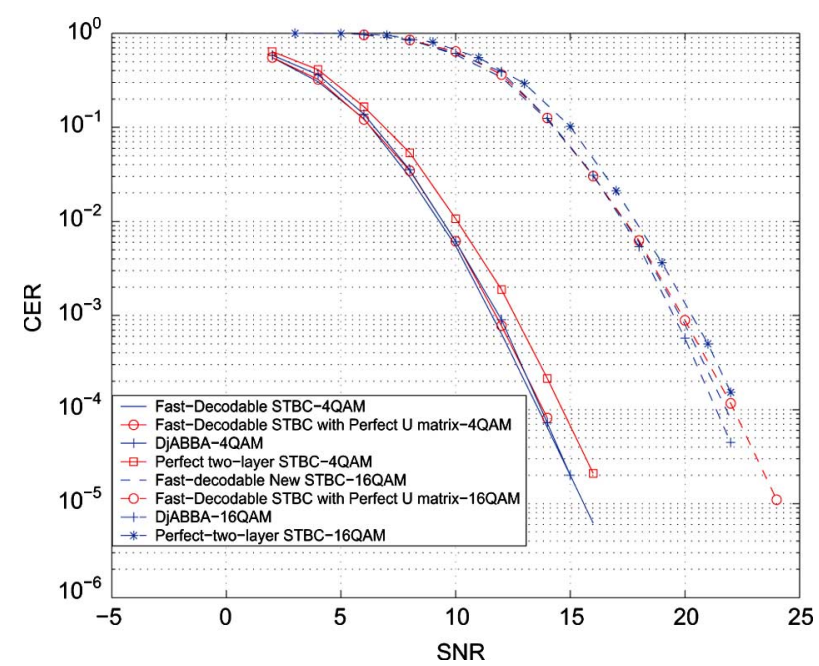

Fig. 2. Comparison of the CER of different $4 \times 2$ STBCs with 4-QAM signaling.

Determinant and multiplicity values are shown in Table II. It can be seen that the proposed $4 \times 2 \mathrm{STBC}$ has the smallest $\sum A(2, \delta)$, when compared to the rank-2 code with perfect rotation matrix $\boldsymbol{U}$ in [19]. The CERs are shown in Fig. 2. The proposed code achieves the best CER up to the CER of $10^{-5}$. Due to the diversity loss, the performance curves of the new code and the one of DjABBA cross over at CER of $2 \times 10^{-5}$.

For 16-QAM signaling, the best matrix $\boldsymbol{U}$ with $N=17$ and $n_{\ell}=3,4,5,13$ is

$$
\left[\begin{array}{rrrr}
0.22+0.44 j & 0.22+0.44 j & 0.22+0.44 j & 0.22+0.44 j \\
0.05+0.50 j & -0.49+0.05 j & -0.05-0.50 & 0.50-0.05 j \\
-0.30-0.40 j & 0.30+0.40 j & -0.30-0.40 j & 0.30+0.40 j \\
0.05-0.50 j & -0.50-0.05 j & -0.05+0.50 j & 0.50+0.05 j
\end{array}\right] .
$$

The performance of this code is compared with that of other codes in Fig. 2. We can see that, at $\mathrm{CER}=10^{-4}$, it requires an SNR $0.4 \mathrm{~dB}$ higher than the best known code of [8], which was not designed for reduced-complexity decoding.

Finally, we notice that the first two colums of $\boldsymbol{X}_{1,2}$ are two stacked Alamouti blocks. This provides the orthogonality condition $\left\langle\boldsymbol{f}_{2}, \boldsymbol{e}_{1}\right\rangle=0$. Therefore, the worst case decoding complexity of fast-decodable $4 \times 2$ STBCs is $2 M^{7}$, as compared to a standard SD complexity $M^{8}$.

\section{CONCLUSION}

We have derived conditions for reduced-complexity ML decoding, and applied them to a unified analysis of two families of full-rate full-diversity $2 \times 2$ STBCs that were recently proposed. Moreover, we have compared their minimum determinant, CER performance, and shaping property, and examined how both families allow low-complexity ML decoding. We have also introduced design criteria of fast-decodable STBCs for $2 \times 2$
MIMO. These design criteria were finally extended to the construction of a fast-decodable $4 \times 2$ code. By combining algebraic and quasi-orthogonal STBC structures, a new code was found that outperforms any known $4 \times 2$ code for 4-QAM signaling, yet with a decoding complexity of $2 M^{7}$ in lieu of the worst case ML decoding complexity $M^{8}$.

\section{ACKNOWLEDGMENT}

The authors are grateful for the constructive comments of the anonymous reviewers.

\section{REFERENCES}

[1] S. M. Alamouti, "A simple transmit diversity technique for wireless communications," IEEE J. Select. Areas Commun., vol. 16, no. 8, pp. 1451-1458, Oct. 1998.

[2] V. Tarokh, H. Jafarkhani, and A. R. Calderbank, "Space-time block codes from orthogonal designs," IEEE Trans. Inf. Theory, vol. 45, no. 5, pp. 1456-1467, Jul. 1999.

[3] V. Tarokh, N. Seshadri, and A. R. Calderbank, "Space-time codes for high data rate wireless communications: Performance criterion and code construction," IEEE Trans. Inf. Theory, vol. 44, no. 2, pp. 744-765, Mar. 1998.

[4] J.-C. Guey, M. P. Fitz, M. R. Bell, and W.-Y. Guo, "Signal design for transmitter diversity wireless communication systems over ranleith fading channels," IEEE Trans. Commun., vol. 47, no. 4, pp. 527-537, Apr. 1999.

[5] H. Jafarkhani, "A quasi-orthogonal space-time block code," IEEE Commun. Lett., vol. 49, no. 1, pp. 1-4, Jan. 2001.

[6] O. Tirkkonen and A. Hottinen, "Square-matrix embeddable space-time block codes for complex signal constellations," IEEE Trans. Inf. Theory, vol. 48, no. 2, pp. 384-395, Feb. 2002.

[7] O. Tirkkonen and R. Kashaev, "Combined information and performance optimization of linear MIMO modulations," in Proc IEEE Int. Symp. Information Theory (ISIT 2002), Lausanne, Switzerland, Jun./Jul. 2002, p. 76.

[8] A. Hottinen and O. Tirkkonen, "Precoder designs for high rate spacetime block codes," in Proc. Conf. Information Sciences and Systems, Princeton, NJ, Mar. 2004.

[9] A. Hottinen, O. Tirkkonen, and R. Wichman, Multi-Antenna Transceiver Techniques for $3 G$ and Beyond. Chichester, U.K.: Wiley, 2003.

[10] J. Paredes, A. B. Gershman, and M. G. Alkhanari, "A $2 \times 2$ space-time code with non-vanishing determinants and fast maximum likelihood decoding," in Proc IEEE Int. Conf. Acoustics, Speech, and Signal Processing (ICASSP 2007), Honolulu, HI, Apr. 2007, pp. 877-880.

[11] M. Samuel and M. P. Fitz, "Reducing the detection complexity by using $2 \times 2$ multi-strata space-time codes," in Proc IEEE Int. Symp. Information Theory (ISIT 2007), Nice, France, Jun. 2007, pp. 1946-1950.

[12] S. Sezginer and H. Sari, "A full-rate full-diversity $2 \times 2$ space-time code for mobile WiMAX systems," in Proc. IEEE Int. Conf. Signal Processing and Communications, Dubai, Jul. 2007.

[13] E. Viterbo and E. Biglieri, "A universal lattice decoder," in GRETSI 14-eme Colloque, Juan-les-Pins, France, Sep. 1993, pp. 391-394.

[14] C. P. Schnorr and M. Euchner, "Lattice basis reduction: Improved practical algorithms and solving subset sum problems," Math Programm., vol. 66, pp. 181-191, 1994.

[15] E. Viterbo and J. Boutros, "A universal lattice code decoder for fading chanel," IEEE Trans. Inf. Theory, vol. 45, no. 5, pp. 1639-1642, Jul. 1999.

[16] M. O. Damen, H. El Gamal, and G. Caire, "On maximum-likelihood detection and the search for the closest lattice point," IEEE Trans. Inf. Theory, vol. 49, no. 10, pp. 2389-2402, Oct. 2003.

[17] F. Oggier and E. Viterbo, "Algebraic number theory and code design for ranleigh fading channels," Foundations and Trends in Communications and Information Theory, vol. 1, pp. 333-415, 2004.

[18] J.-C. Belfiore, G. Rekaya, and E. Viterbo, "The golden code: A $2 \times 2$ full-rate space-time code with non-vanishing determinants," IEEE Trans. Inf. Theory, vol. 51, no. 4, pp. 1432-1436, Apr. 2005.

[19] F. Oggier, G. Rekaya, J.-C. Belfiore, and E. Viterbo, "Perfect space-time block codes," IEEE Trans. Inf. Theory, vol. 52, no. 9, pp. 3885-3902, Sep. 2006.

[20] Y. Hong, E. Viterbo, and J.-C. Belfiore, "A space-time block coded multiuser MIMO downlink transmission scheme," in Proc. IEEE Int. Symp. Information Theory, Seattle, WA, Jun./Jul. 2006, pp. 257-261. 
Ezio Biglieri (M'73-SM'82-F' 89) was born in Aosta (Italy). He received his formal training in electrical engineering at Politecnico di Torino (Torino, Italy), where he received the Dr. Engr. degree in 1967.

$\mathrm{He}$ is presently an Adjunct Professor of Electrical Engineering at the University of California, Los Angeles (UCLA) and an honorary Professor at Universitat Pompeu Fabra, Barcelona, Spain. Previously, he was a Professor at the University of Napoli (Napoli, Italy), at Politecnico di Torino, and at the University of California, Los Angeles (UCLA). He has held visiting positions with the Department of System Science, UCLA; the Mathematical Research Center, Bell Laboratories, Murray Hill, NJ; Bell Laboratories, Holmdel, NJ; the Department of Electrical Engineering, UCLA, the Telecommunication Department of The Ecole Nationale Supérieure des Télécommunications, Paris, France; the University of Sydney, Australia; the Yokohama National University, Japan; the Electrical Engineering Department of Princeton University, Princeton, NJ; the University of South Australia, Adelaide; the University of Melbourne, Australia; the Institute for Communications Engineering, Munich Institute of Technology, Germany; the Institute for Infocomm Research, National University of Singapore; the National Taiwan University, Taipei, Republic of China; the University of Cambridge, U.K.; and ETH Zurich, Switzerland.

Prof. Biglieri was elected three times to the Board of Governors of the IEEE Information Theory Society, and he served as its President in $1999 . \mathrm{He}$ is currently the Editor-in-Chief of the IEEE TRANSACTIONS ON INFORMATION THEORY and of the Journal of Communications and Networks. Among other honors, in 2000 he received the IEEE Third-Millennium Medal and the IEEE Donald G. Fink Prize Paper Award, in 2001 the IEEE Communications Society Edwin Howard Armstrong Achievement Award, and a Best Paper Award from WPMC'01, Aalborg, Denmark, and in 2004 the Journal of Communications and Networks Best Paper Award.

Yi Hong (S'00-M'05) received the Ph.D. degree in electrical engineering and telecommunications from the University of New South Wales (UNSW), Sydney, Australia, in 2004.

From 2004 to 2007, she was a Postdoctoral Fellow at the Institute for Telecommunications Research (ITR), University of South Australia (UniSA),
Australia. She is currently a Lecturer on Wireless Communications at the Institute of Advanced Telecom., University of Wales, Swansea U.K. Her research interests are communication and information theory, and signal processing.

Dr. Hong received the Early Career Researcher Best Paper Award at the 2007 Australian Communication Theory workshop. She is a member of the ARC Communications Research Network (ACoRN).

Emanuele Viterbo (M'95-SM'04) was born in Torino, Italy, in 1966. He received the Laurea degree in electrical engineering in 1989 and the Ph.D. degree in electrical engineering in 1995, both from the Politecnico di Torino, Torino, Italy.

From 1990 to 1992, he was with the European Patent Office, The Hague, The Netherlands, as a patent examiner in the field of dynamic recording and error-control coding. Between 1995 and 1997, he held a postdoctoral position in the Dipartimento di Elettronica of the Politecnico di Torino in Communications Techniques over Fading Channels. He became Associate Professor at Politecnico di Torino, Dipartimento di Elettronica in 2005 and since November 2006 has been Full Professor in DEIS at Università della Calabria, Rende, Italy. He held many Visiting Researcher appointments: In 1993, in the Communications Department of DLR, Oberpfaffenhofen, Germany; Iin 1994 and 1995, at E.N.S.T., Paris, France; in 1998, in the Information Sciences Research Center of AT\&T Research, Florham Park, NJ; in 2003, at the Mathematics Department of EPFL, Lausanne, Switzerland; n 2004, at the Telecommunications Department of UNICAMP, Campinas, Brazil; and in 2005, at the ITR of UniSA, Adelaide, Australia. His main research interests are in lattice codes for the Gaussian and fading channels, algebraic coding theory, algebraic space-time coding, digital terrestrial television broadcasting, and digital magnetic recording

Dr. Viterbo was awarded a NATO Advanced Fellowship in 1997 from the Italian National Research Council. He is Associate Editor of IEEE TRANSACTIONS ON INFORMATION THEORY, the European Transactions on Telecommunications and Journal of Communications and Networks. 